\title{
A THEORETICAL INVESTIGATION OF THE EFFECT OF THE STOCHASTICITY IN THE MATERIAL PROPERTIES ON THE CHATTER DETECTION DURING TURNING
}

\author{
Henrik T. Sykora \\ Department of Applied Mechanics \\ Faculty of Mechanical Engineering \\ Budapest University of Technology and Economics \\ Budapest, Hungary \\ Email: sykora@mm.bme.hu
}

\author{
Daniel Bachrathy \\ Gabor Stepan \\ Department of Applied Mechanics \\ Faculty of Mechanical Engineering \\ Budapest University of Technology and Economics \\ Budapest, $\mathrm{H}-1111$ \\ Hungary \\ bachrathy@mm.bme.hu \\ stepan@mm.bme.hu
}

\begin{abstract}
In this work the effect of the inhomogeneous material properties are investigated in regenerative turning processes by introducing white noise in the cutting coefficient. The model is a one degree of freedom linear delayed oscillator with stochastic parameters. A full discretization method is used to calculate the time evolution of the second moment to determine the moment stability of the turning process. The resultant stability chart is compared with the deterministic turning model.
\end{abstract}

\section{NOMENCLATURE}

$\kappa$ Dimensionless cutting force coefficient

$\sigma$ Relative deviation of the dimensionless cutting force coefficient

$\zeta$ Damping coefficient

$\tilde{t}, \tilde{\tau} \quad$ Time and time delay

$t, \tau$ Dimensionless time and time delay

$\tilde{y}_{\tilde{t}} \quad$ Time function of the displacement of the tool

$y_{t}$ Dimensionless displacement of the tool

$\Gamma_{\tilde{t}}$ Langevin-force

$W_{t} \quad$ Wiener process on the dimensionless time-scale

\section{INTRODUCTION}

In the field of manufacturing science machine tool vibration is source of many problems since decades $[1,2]$. The commonly used models involve deterministic delay differential equations, in which the parameters are usually considered to be constant [3-8]. During the measurements of these parameters, the average is considered and the variance is attributed to the quality of the measurement. However, it is easy to see that materials are not homogeneous, so variance can be an inherent property, which leads us to the use of stochastic models. A typical measured cutting force signal of an orthogonal planning process (material: $\mathrm{AL}$ 2024 T351) is shown in Fig. 1, where the time signal is measured by Kistler dynamometer (9129AA), with the cutting parameters chip width $w=1 \mathrm{~mm}$, chip thickness $h_{0}=0.1 \mathrm{~mm}$, feed rate $v_{\mathrm{f}}=10000 \mathrm{~mm} / \mathrm{min}$ and rake angle $\alpha_{\mathrm{r}}=5^{\circ}$. All the mainblue), the feed- ( $\square$ orange) and the passive- ( $\square$ red) force components have a significant variation around their mean value under the range where the transient seem to be settled ( $\square$ green area in Fig. 1). On the histograms of these stationary time signals marked with $\square$ green, one can observe that the signal shows a Gaussian distribution as shown on Fig. 2, with the help of fitted normal distributions. In this test case for the main force component a large relative variance $\left(\sigma_{0}=0.089\right)$ is observed. This can be related to different physical phenomena, like shear-plane 


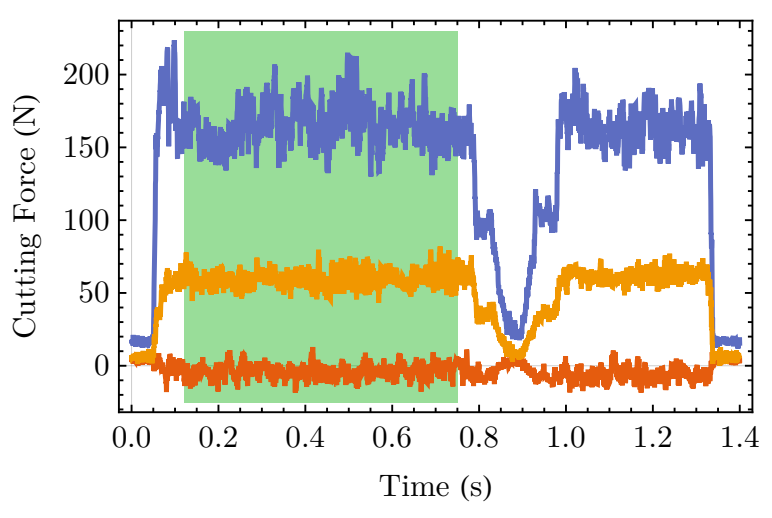

FIGURE 1: TIME SIGNAL OF A CUTTING FORCE MEASUREMENT DURING TURNING: THE — MAIN-, THE FEED- AND THE PASSIVE FORCE COMPONENTS ARE ALL SHOWING STOCHASTIC BEHAVIOUR

oscillation [9] or inhomogeneity in the texture structure. These are very complex processes, thus a stochastic noise excitation is used to take the effect of these unmodelled dynamics into account. Further advantages of this stochastic models are, that it can predict the noise of the measured signals, which is typically important near the stability borders, where the signs of chattervibration already appears in the stable parameter domain.

\section{MATHEMATICAL MODEL}

\section{The Mechanical Model}

To investigate the effect of stochasticity, the a simple one degree of freedom regenerative model of turning is used [1,2]:

$$
\ddot{\tilde{y}_{\tilde{t}}}+2 \zeta \omega_{\mathrm{n}} \dot{\tilde{y}_{\tilde{t}}}+\omega_{\mathrm{n}}^{2} \tilde{y}_{\tilde{t}}=\frac{F_{c}\left(h_{\tilde{t}}\right)}{m}
$$

where $\omega_{\mathrm{n}}=\sqrt{c / m}$ is the natural frequency, $\zeta=b /\left(2 m \omega_{\mathrm{n}}\right)$ is the damping coefficient, and $c, b, m$ are the resultant stiffness, the resultant damping and the modal mass, respectively (see Fig. 3). To analyze the small amplitude vibration around the stationary position a widely used linear cutting force model is considered $[1-5,10]$ :

$$
F_{c}\left(h_{\tilde{t}}\right)=k_{1}\left(1+\sigma_{0} \Gamma_{\tilde{t}}\right) w h_{\tilde{t}},
$$

where $k_{1}$ is the linear cutting force coefficient, $w$ is the chip width and $h_{\tilde{t}}$ is the chip thickness. This force model can be used without the loss of generality for linear stability calculations, since the $k_{1}$ linear cutting force coefficient can be determined by means of Taylor expansion around the $h_{0}$ nominal chip thickness even
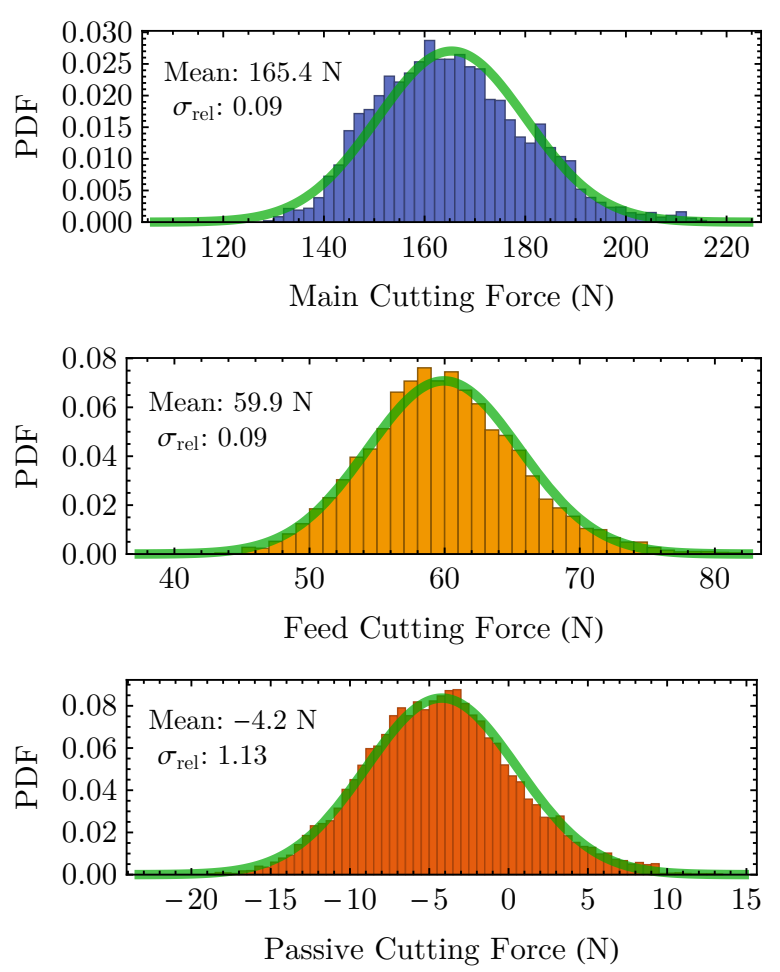

FIGURE 2: THE HISTOGRAM OF THE MEASURED FORCE SIGNALS. THE HISTOGRAMS SHOW A NEAR-GAUSSIAN DISTRIBUTION COMPARED WITH THE $\square$ THEORETICAL CURVES OF THE NORMAL DISTRIBUTION

for nonlinear force characteristics [11]. To consider the fluctuations in the cutting force, a $\Gamma_{\tilde{t}}$ Gaussian white-noise process, called Langevin force is introduced in the force model. The $\Gamma_{t}$ Gaussian white-noise process is independent of the position of the tool, and has a constant power spectrum $[12,13]$. To describe the regenerative effect, the $h_{\tilde{t}}$ chip thickness is calculated using the actual and a delayed tool position [14]:

$$
h_{\tilde{t}}=h_{0}-\tilde{y}_{\tilde{t}}+\tilde{y}_{\tilde{t}+\tilde{\tau}} .
$$

\section{The Stochastic Dimensionless Equation for Turning}

To investigate the effect of the stochasticity in the material properties the dimensionless form of the one degree of freedom model of the turning process can be used without the loss of generality.

$$
\ddot{y}_{t}+2 \zeta \dot{y}_{t}+y_{t}=\kappa\left(1+\sigma \Gamma_{t}\right)\left(1-y_{t}+y_{t-\tau}\right),
$$




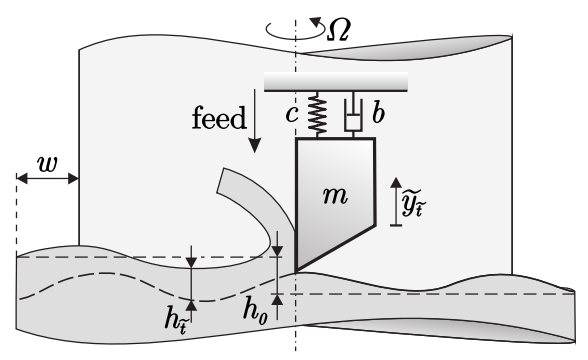

FIGURE 3: ONE DEGREE OF FREEDOM MECHANICAL MODEL FOR TURNING

where

$$
\begin{gathered}
t=\omega_{\mathrm{n}} \tilde{t}, \quad y_{t}=\frac{\tilde{y}_{t}}{h_{0}}, \quad \kappa=\frac{k_{1} w}{m \omega_{\mathrm{n}}^{2}}, \\
\sigma=\frac{\sigma_{0}}{\sqrt{\omega_{\mathrm{n}}}}, \quad \int_{t}^{t+\Delta t} \Gamma_{s} \mathrm{~d} s=\Delta W_{t} .
\end{gathered}
$$

The $\sqrt{\omega_{\mathrm{n}}}$ in the denominator of $\sigma$ in equation (5)-(6) comes from the transformation of the time scale of the $\Gamma_{\tilde{t}}$ white noise process. When changing time scales the intensity of the white noise has to be adjusted:

$$
\Gamma_{\tilde{t}}=\frac{1}{\sqrt{\omega_{\mathrm{n}}}} \Gamma_{t} .
$$

To model the Gaussian white noise the Wiener process is used. Its increment has the expected value $\mathbb{E}\left(\mathrm{d} W_{t}\right)=0$, the variance $\mathbb{E}\left(\mathrm{d} W_{t}^{2}\right)=\mathrm{d} t$, and the increments are independent, thus $\mathbb{E}\left(\mathrm{d} W_{t} \mathrm{~d} W_{s}\right)=0$ for $s \neq t$. The process itself has the time dependent normal distribution of $W_{t} \sim \mathscr{N}\left(0, \sigma^{2}=t\right)$.

If the expected value is taken of both sides of the equation (4), an $x_{t}$ perturbation process can be introduced around the $\mathbb{E}\left(y_{s t}\right)=\kappa$ equilibrium point of the equation, where $\mathbb{E}\left(x_{t}\right)=0$, as shown in (8).

$$
y_{t}=\mathbb{E}\left(y_{s t}\right)+x_{t} .
$$

Substituting back (8) into (4) the evolution equation for the $x_{t}$ perturbation process is gained:

$$
\ddot{x}_{t}+2 \zeta \dot{x}_{t}+x_{t}=\kappa\left(1+\sigma \Gamma_{t}\right)\left(x_{t-\tau}-x_{t}\right)+\kappa \sigma \Gamma_{t} .
$$

Note, on the right hand side of (9) both multiplicative and additive noise are present. form:

Equation (9) can be rewritten into a first order increment

$$
\mathrm{d} \mathbf{x}_{t}=\left(\boldsymbol{\alpha} \mathbf{x}_{t}+\boldsymbol{\beta} \mathbf{x}_{t-\tau}\right) \mathrm{d} t+\left(\boldsymbol{\sigma}_{\boldsymbol{\alpha}} \mathbf{x}_{t}+\boldsymbol{\sigma}_{\boldsymbol{\beta}} \mathbf{x}_{t-\tau}+\boldsymbol{\sigma}\right) \mathrm{d} W_{t},
$$

where

$$
\begin{array}{cc}
\mathbf{x}_{t}=\left(\begin{array}{l}
x_{t} \\
\dot{x}_{t}
\end{array}\right), \quad \boldsymbol{\alpha}=\left(\begin{array}{cc}
0 & 1 \\
-(1+\kappa) & -2 \zeta
\end{array}\right), \quad \boldsymbol{\beta}=\left(\begin{array}{ll}
0 & 0 \\
\kappa & 0
\end{array}\right), \\
\boldsymbol{\sigma}_{\boldsymbol{\alpha}}=\left(\begin{array}{cc}
0 & 0 \\
-\kappa \sigma & 0
\end{array}\right), \quad \boldsymbol{\sigma}_{\boldsymbol{\beta}}=\left(\begin{array}{cc}
0 & 0 \\
\kappa \sigma & 0
\end{array}\right), \quad \boldsymbol{\sigma}=\left(\begin{array}{c}
0 \\
\kappa \sigma
\end{array}\right) .
\end{array}
$$

If the equation system is in the form shown in (10) a fulldiscretization method [15] can be used to rewrite it to a discreet mapping:

$$
\mathbf{X}_{n+1}=\mathbf{A} \mathbf{X}_{n}+\mathbf{B} \mathbf{X}_{n}+\mathbf{c}
$$

where the 2(N+1)-element state vector $\mathbf{X}_{n}$ contains the discretized solution of $\mathbf{x}_{t}$ over the interval $\left[t_{n}-\tau, t_{n}\right]$ with $t_{n}=n \tau / N$. The A matrix contains the discretized infinitesimal operator for the drift (deterministic) part of the system, while $\mathbf{B}$ contains the multiplicative and $\mathbf{c}$ the additive component of the diffusion (stochastic) part.

\section{Moment Stability}

The discrete mapping for the first two moments can be written using (13):

$$
\begin{gathered}
\mathbb{E}\left(\mathbf{X}_{n+1}\right)=\mathbf{A} \mathbb{E}\left(\mathbf{X}_{n}\right), \\
\mathbb{E}\left(\mathbf{X}_{n+1} \mathbf{X}_{n+1}^{\top}\right)=\mathbb{E}\left((\mathbf{A}+\mathbf{B}) \mathbf{X}_{n} \mathbf{X}_{n}^{\top}(\mathbf{A}+\mathbf{B})^{\top}\right)+\ldots
\end{gathered}
$$

where the neglected components are independent of the second moment.

In the equation (15) the two side multiplications can be rewritten to a single matrix multiplication, if the elements of the $\mathbb{E}\left(\mathbf{X}_{n} \mathbf{X}_{n}^{\top}\right)$ autocorrelation matrix are arranged into a vector according to the following form, satisfying the properties of the expected value of $\mathbf{A}$ and $\mathbf{B}$.

$$
\mathbb{E}\left((\mathbf{A}+\mathbf{B})\left(" \mathbf{M}^{\prime \prime}\right)(\mathbf{A}+\mathbf{B})^{\top}\right) \rightarrow(\widetilde{\mathbf{A}}+\mathbb{E}(\widetilde{\mathbf{B}}))\left(" \mathbf{v}^{\prime}\right)
$$

In equation (16) "M" denotes a symmetric matrix and "v" denotes the vector of the independent elements of the "M" matrix. The $\lambda$ spectral radius of the $(\widetilde{\mathbf{A}}+\mathbb{E}(\widetilde{\mathbf{B}}))$ infinitesimal generator matrix determines the stability of the second moment while 
assuring the stability of the first moment: if $\lambda<1$, then the second moment is stable, if $\lambda>1$ then it is unstable [15].

In case of the existence of the stable, stationary solution, the stationary second moment can be calculated by the formula [15]:

$$
\mathbf{v}^{*}=(\mathbf{I}-(\widetilde{\mathbf{A}}+\mathbb{E}(\widetilde{\mathbf{B}}))) \widetilde{\mathbf{c}}
$$

For the detailed derivation of $\mathbf{X}_{n}, \mathbf{A}, \mathbf{B}, \mathbf{c}$ and the infinitesimal generator matrices for the second moment see [15].

\section{TEST CASE}

To analyze the effect of the stochastic material properties for the stability of the turning process, a stability chart was calculated by the methods described above for a selected parameters: $\sigma=0.1$ and $\zeta=0.1$. The obtained stable area denoted with color $\square$ dark blue in Fig. 4. To see effect of the noise, the stability boundary for the deterministic case is plotted with dashed line, according to the analytical solution defined by the following parametric curves [16]:

$$
\begin{aligned}
\kappa(\omega) & =\frac{2 \zeta^{2} \omega^{2}}{\omega^{2}-1}+\frac{\omega^{2}-1}{2} \\
\tau(\omega, m) & =\frac{2}{\omega}\left(\tan ^{-1}\left(\frac{1-\omega^{2}}{2 \zeta \omega}\right)+m \pi\right),
\end{aligned}
$$

where $\omega$ is the vibration (chatter) frequency along the stability boundary and $m$ is the lobe number.

Altough the change in the linear stability boundary is insignificant, the method predicts large amplitude vibrations near the stability boundary, presented by the stationary second moment above the stability chart for $\kappa=0.3$. These theoretical predictions are valid only for small amplitude vibration, due to the nonlinear cutting force characteristics [17], and the fly-over effect [11]. The solutions with large second moment can jump into a large amplitude chaotic attractor, which is the chatter-vibration, and because of this phenomena the theoretical stability boundary cannot be reached in practice [18]. It means that the measured stability boundaries would shift towards smaller chip width values due this stochastic effect. To present this effect amplitude limits are showed at $\sigma_{x, s t}=1$ and $2 \sigma_{x, s t}=1$ on Fig. 4 , where the amplitude of the tool position is smaller than $h_{0}$ with $68.3 \%$ and $95.45 \%$ probability respectively $\left(\sigma_{x, s t}\right.$ denotes the first element of the stationary second moment vector $\mathbf{v}^{*}$ in equation (17)). This comes from the fact that the stationary distribution of the solution of equation (9) is also Gaussian distritubion. It is important to note, that even the presence of a small additive noise through the cutting force makes this type of resonance phenomena significant [19]. Due to this effect the measurements between the lobes can show a large amplitude vibration, despite of the system being asymptotically stable.

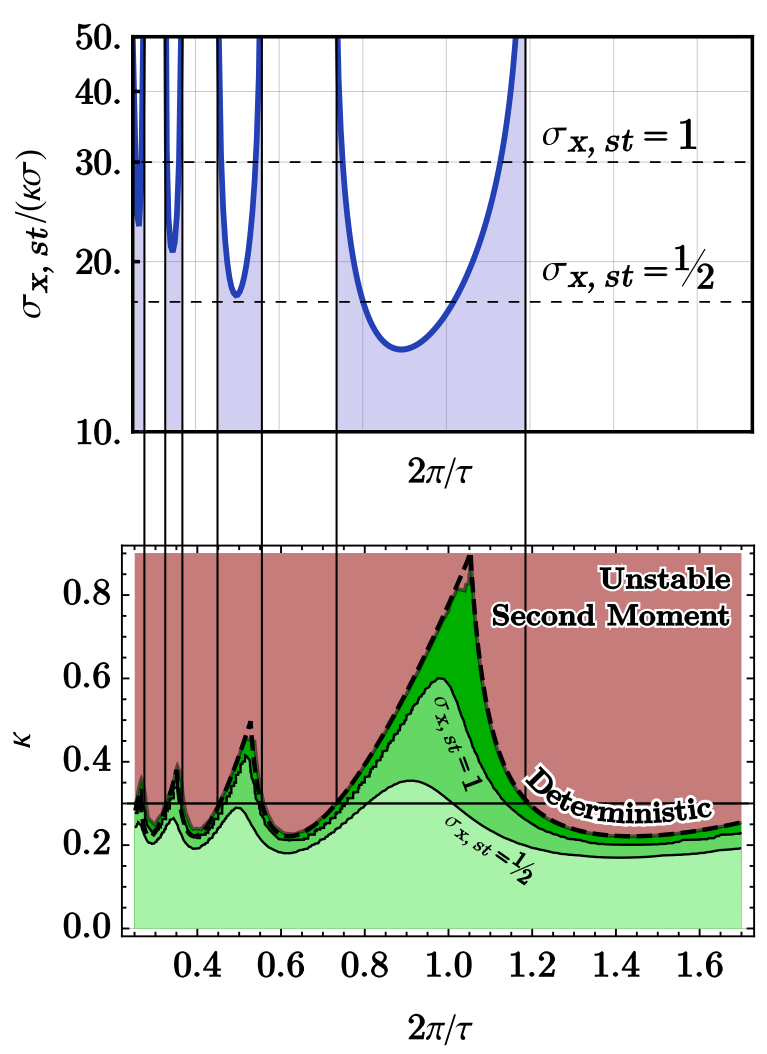

FIGURE 4: SECOND MOMENT (SM) RATIO OF THE STATIONARY SOLUTION ALONG $\kappa=0.3, \sigma=0.1$ AND STABILITY MAP OF THE SECOND MOMENT FOR THE SAME NOISE INTENSITY.

\section{CONCLUSION}

In the present study first we show that the cutting force should be modeled as a stochastic process, driven by a Gaussian white noise. Furthermore a possible explanation is given for the measurement difficulties $[20,21]$ of the theoretically predicted stability boundaries with the use of a stochastic model, but this needs to be validated through a series of orthogonal turning tests. The applied full discretization method gives a good approximation for high discretization resolution, due to the very slow convergence of the spectral radius of the second moment generator matrices. An additional problem is that the computational complexity is proportional to the 4-th order of the number of discretized points, which leads to high computational time. Thus an important future task is to further improve the convergence of the applied method.

\section{REFERENCES}

[1] Tlusty, J., and Spacek, L., 1954. Self-excited vibrations on machine tools. 
[2] Tobias, S., 1965. Machine-tool Vibration.

[3] Insperger, T., Mann, B. P., Surmann, T., and Stépán, G., 2008. "On the chatter frequencies of milling processes with runout". International Journal of Machine Tools and Manufacture, 48(10), aug, pp. 1081-1089.

[4] Insperger, T., Stépán, G., Bayly, P., and Mann, B., 2003. "Multiple chatter frequencies in milling processes". Journal of Sound and Vibration, 262(2), apr, pp. 333-345.

[5] Insperger, T., 2002. "Stability analysis of periodic delaydifferential equations modeling machine tool chatter". PhD thesis.

[6] Chełminski, K., Hömberg, D., and Rott, O., 2011. "On a thermomechanical milling model". Nonlinear Analysis: Real World Applications, 12(1), feb, pp. 615-632.

[7] Insperger, T., 2010. "Full-discretization and semidiscretization for milling stability prediction: Some comments". International Journal of Machine Tools and Manufacture, 50(7), jul, pp. 658-662.

[8] Khasawneh, F. A., and Munch, E., 2016. "Chatter detection in turning using persistent homology". Mechanical Systems and Signal Processing, 70-71, mar, pp. 527-541.

[9] Pálmai, Z., and Csernák, G., 2009. "Chip formation as an oscillator during the turning process". Journal of Sound and Vibration, 326(3-5), oct, pp. 809-820.

[10] Altintas, Y., 2011. Manufacturing Automation, Vol. 227. Cambridge University Press, Cambridge.

[11] Dombovari, Z., Barton, D. A., Eddie Wilson, R., and Stepan, G., 2011. "On the global dynamics of chatter in the orthogonal cuttingmodel”. International Journal of NonLinear Mechanics, 46(1), jan, pp. 330-338.

[12] Wedig, W. V., 2005. "Vertical dynamics of riding cars under stochastic and harmonic base excitations". Solid Mechanics and its Applications, 122(2), pp. 371-381.

[13] Wedig, W. V., 2011. "Stochastic parameter resonance of road-vehicle systems and related bifurcation problems". Solid Mechanics and its Applications, 29(2), pp. 221-228.

[14] Stépán, G., 1989. Retarded dynamical systems: stability and characteristic functions.

[15] Elbeyli, O., Sun, J. Q., and Ünal, G., 2005. "A semidiscretization method for delayed stochastic systems". Communications in Nonlinear Science and Numerical Simulation, 10(1), pp. 85-94.

[16] Lingala, N., Sri Namachchivaya, N., O’Reilly, O. M., and Wihstutz, V., 2013. "Almost sure asymptotic stability of an oscillator with delay feedback when excited by finite-state Markov noise". Probabilistic Engineering Mechanics, 32, apr, pp. 21-30.

[17] BUCKWAR, E., KUSKE, R., L'ESPERANCE, B., and SOO, T., 2006. "NOISE-SENSITIVITY IN MACHINE TOOL VIBRATIONS". International Journal of Bifurcation and Chaos, 16(08), aug, pp. 2407-2416.

[18] Kuske, R., 2006. "Multiple-scales approximation of a co- herence resonance route to chatter". Computing in Science \& Engineering, 8(3), may, pp. 35-43.

[19] Kuske, R., 2010. "Competition of Noise Sources in Systems with Delay: The Role of Multiple Time Scales". Journal of Vibration and Control, 16(7-8), jun, pp. 983-1003.

[20] Schmitz, T. L., 2003. "Chatter recognition by a statistical evaluation of the synchronously sampled audio signal [2]". Journal of Sound and Vibration, 262(3), pp. 721-730.

[21] Altintas, Y., and Chan, P. K., 1992. "In-process detection and suppression of chatter in milling". International Journal of Machine Tools and Manufacture, 32(3), pp. 329347.

\section{ACKNOWLEDGMENT}

The research leading to these results has received funding from the European Research Council under the European Union's Seventh Framework Programme (FP7/2007-2013) ERC Advanced grant agreement No340889 and it was supported by the Hungarian Scientific Research Fund (OTKA PD-112983) and the Janos Bolyai Research Scholarship of the Hungarian Academy of Sciences. 